\title{
Asociaciones de poliquetos bentónicos y su relación con los parámetros sedimentarios en el sur de la Bahía Samborombón (provincia de Buenos Aires, Argentina)
}

\author{
Pablo E. Denuncio ${ }^{1, \varpi}$; María E. Trassens ${ }^{1,2}$; Delfina M.P. Cantatore ${ }^{1}$; Juan P. Lancia ${ }^{1,2}$ \\ \& RICARDO O. BASTIDA ${ }^{1}$
}

1. Instituto de Investigaciones Marinas y Costeras (IIMyC-CONICET), Facultad de Ciencias Exactas y Naturales, Universidad Nacional de Mar del Plata, Argentina. 2. Instituto de Geología de Costas y del Cuaternario, Facultad de Ciencias Exactas y Naturales, Universidad Nacional de Mar del Plata, Mar del Plata, Argentina.

\begin{abstract}
RESUMEN. El presente estudio describe la estructura de las asociaciones de poliquetos bentónicos del extremo sur de la Bahía de Samborombón, y su relación con los sedimentos, dos décadas después del último estudio realizado en la zona. Se establecieron tres estaciones de muestreo: Punta Rasa, Tapera de López y Arroyo San Clemente. En las mismas fueron identificadas cuatro especies de poliquetos: Laeonereis culveri, Heteromastus similis, Alitta succinea y Polydora cornuta, de las cuales L. culveri y H. similis resultaron las más abundantes. Se detectaron variaciones estacionales en la asociación y la abundancia de poliquetos en relación a la granulometría del sedimento. Punta Rasa y Tapera de López estuvieron caracterizadas casi exclusivamente por arena fina; allí se observaron las cuatro especies de poliquetos, siendo $H$. similis el dominante. En el Arroyo San Clemente, el sedimento dominante fue la arena media, adquiriendo gran importancia las pelitas. Alitta succinea estuvo ausente y Laeonereis culveri fue dominante. Estos resultados sugieren que los parámetros sedimentarios influirían sobre la variación en la distribución de poliquetos y que dicha variación se puede presentar aun en una escala espacial pequeña. Tanto la riqueza específica de poliquetos como la densidad general (individuos $/ \mathrm{m}^{2}$ ) por estación fueron menores a los valores anuales observados en los estudios efectuados en la década del noventa. Esto podría deberse a que el presente trabajo fue realizado a principio de otoño, luego de la concentración de aves playeras (primavera-verano), que depredan con intensidad a estos organismos bentónicos. Este tipo de estudios resulta importante al tratarse de un ambiente potencialmente afectado por las actividades antrópicas que tienen lugar en la zona interna del estuario del Río de la Plata.
\end{abstract}

[Palabras clave: Laeonereis culveri, Heteromastus similis, Alitta succinea, Polydora cornuta, asociaciones bentónicas, sedimentos, estuario del Río de la Plata]

\begin{abstract}
AвSTRACT. Benthic polychaete association and their relationship with sedimentary parameters at south of Samborombón Bay (Buenos Aires province, Argentina): The present work describes the structure of benthic polychaete association of the southermost part of Samborombón Bay and its relationship with sediment after two decades of the last study made in the same area. Three sampling stations were established: Punta Rasa, Tapera de López and Arroyo San Clemente. Four species of polychaetes were identified: Laeonereis culveri, Heteromastus similis, Alitta succinea and Polydora cornuta, being L. culveri and H. similis the most abundant. Variations between stations in terms of the association and relative abundances of polychaetes with granulometry of sediment were recognized. Punta Rasa and Tapera de López were characterized almost exclusively by fine sand, observing the four species of polychaetes with dominance of $H$. similis. In Arroyo San Clemente the dominant sediment was medium sand, with pelites becoming important; A. succinea was absent and L. culveri was dominant. This indicates that variation in the distribution of polychaetes would be strongly influenced by the sedimentary parameters, and that this variation can arise even at small spatial scale. Both, polychaete species richness and general density (individuals $/ \mathrm{m}^{2}$ ) by station were lower than density annual values observed in studies in the ' $90 \mathrm{~s}$. This could be because this study was conducted in the early fall, after shorebirds concentration (spring-summer), that strongly fed of these benthic organisms. This kind of studies is important since it is an environment potentially affected by human activities, taking place mainly in the inner zone of the Río de la Plata estuary.
\end{abstract}

[Keywords: Laeonereis culveri, Heteromastus similis, Alitta succinea, Polydora cornuta, benthic association, sedimentology, Río de la Plata estuary]

\section{INTRODUCCIÓN}

Las costas de la provincia de Buenos Aires se encuentran caracterizadas por dos tipos de ambientes en los que predominan los

\section{Editor asociado: Fernado Unrein}

$\triangle$ pdenunci@mdp.edu.ar fondos blandos: playas arenosas típicamente marinas y planicies areno-fangosas de ambientes mixohalinos (Martin 2002). Las primeras, vinculadas con el ecosistema marino costero, se extienden desde el sur de la Bahía

Enviado: 22 de noviembre de 2014, Fin de arbitraje: 16 de enero de 2015, Última versión revisada: 10 de marzo, Aceptado: 20 de marzo. 
Samborombón, presentan una pendiente suave y alta energía hídrica, y predominan las arenas medianas y gruesas con presencia de bioclastos (Ieno \& Bastida 1998).

Los ambientes mixohalinos incluyen biotopos como los estuarios y albúferas, generalmente con una gran variación en la salinidad (Ringuelet 1962). La Bahía Samborombón es un ambiente mixohalino de baja energía, escasa profundidad, extensas planicies intermareales de baja pendiente y sedimentos areno-fangosos (Martin 2002). Pertenece a la margen externa del estuario del Río de la Plata, que forma parte de la cuenca más importante de Argentina (Urien 1966; Ieno \& Bastida 1998).

En la provincia de Buenos Aires se han llevado a cabo numerosos estudios sobre las comunidades bentónicas de fondos blandos de ambientes mixohalinos, como la laguna costera de Mar Chiquita (Olivier et al. 1972; Ieno \& Elías 1995; Schwindt \& Iribarne 2000), el estuario del puerto del río Quequén (López Gappa et al. 2001) y la Bahía Samborombón (Ieno \& Bastida 1998; Martin et al. 2004). En todos ellos, la fauna se caracteriza por una riqueza específica baja, siendo los poliquetos los organismos más representados.

En estos ambientes mixohalinos, los poliquetos juegan un rol ecológico clave, ya sea por el uso de la materia orgánica depositada en los sedimentos (Martin 2002) o como ítems tróficos de diversas especies de aves y peces marinos (Ieno et al. 2004). Esto implica una transferencia de energía hacia niveles tróficos más altos, compuestos por varias especies de peces, aves y mamíferos marinos (Rodríguez et al. 2002; Botto et al. 2011).
Las investigaciones sobre la relación de los sedimentos y las comunidades bentónicas en este tipo de ambientes resultan útiles para evaluar y monitorear el impacto ambiental (Muniz et al. 2012), en especial en regiones industrializadas, con alta densidad de población y sujetas a una gran presión pesquera (e.g., el estuario del Río de la Plata y, en particular, la Bahía Samborombón). El objetivo del presente trabajo fue caracterizar las asociaciones de poliquetos bentónicos y analizar la relación con los parámetros sedimentarios de las planicies del intermareal del sur de la Bahía Samborombón y canales internos.

\section{MATERIALES Y MÉTODOS}

\section{Área de estudio}

La zona de estudio forma parte del sitio RAMSAR de la Bahía Samborombón, uno de los 21 sitios establecidos para la Argentina. La Bahía Samborombón comprende una línea de costa de $\sim 180 \mathrm{~km}$, e incluye zonas costeras de aguas someras con una profundidad entre 2 y 5 m (Martin 2002). Presenta una zona intermareal muy extensa, de costas bajas y anegadizas, con sustratos blandos constituidos por arcilla, limo y arena predominantemente fina que forman fangos característicos (Urien 1966).

El presente estudio fue llevado a cabo a principio del otoño de 2011 en tres estaciones de muestreo en el extremo sureste de la bahía: Punta Rasa (PR) (36 $\left.17^{\prime} 46.23^{\prime \prime} \mathrm{S}-56^{\circ} 46^{\prime} 37.58^{\prime \prime} \mathrm{O}\right)$, Tapera de López (TL) $\left(36^{\circ} 19^{\prime} 14.33^{\prime \prime} \mathrm{S}-56^{\circ} 46^{\prime} 27.29^{\prime \prime} \mathrm{O}\right)$ y Arroyo San Clemente (SC) $\left(36^{\circ} 20^{\prime} 24.87^{\prime \prime} \mathrm{S}-56^{\circ} 45^{\prime} 07.55^{\prime \prime}\right.$ O) (Figura 1). Punta Rasa es una barra arenosa que marca el límite sur de la Bahía Samborombón, con orientación noroeste y la estación de muestreo

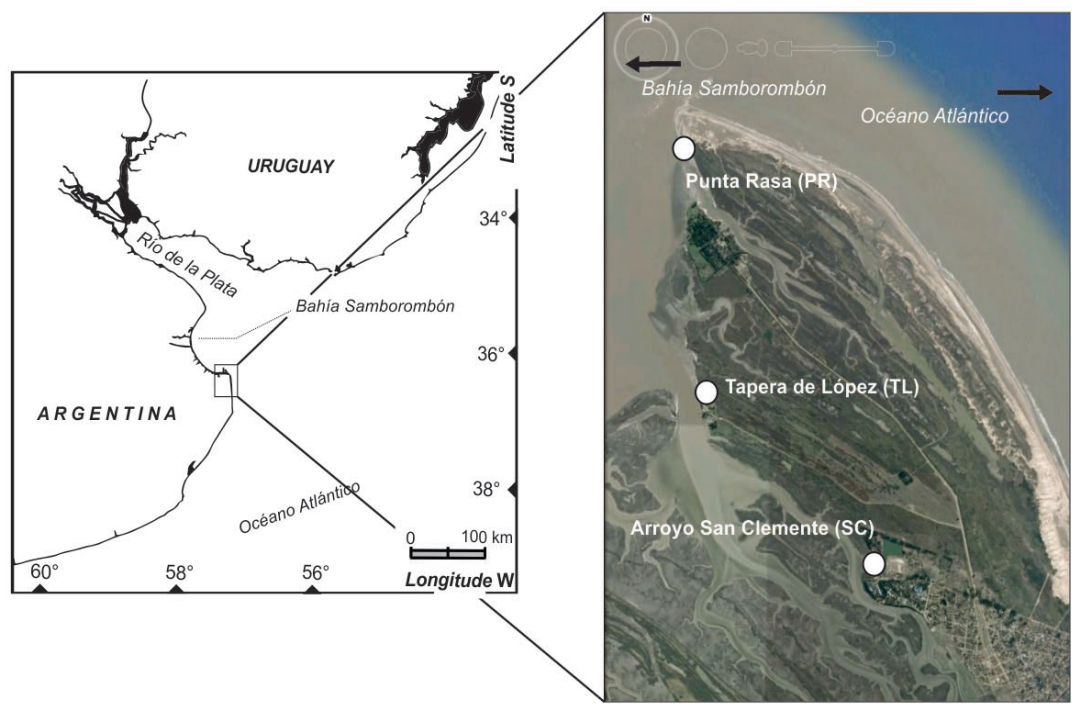

Figura 1. Área de estudio en el sur de la Bahía Samborombón (Argentina), señalando las tres estaciones de muestreo (imagen de Google Earth).

Figure 1. Study area at south of Samborombón Bay (Argentina), indicating the three sampling stations (image from Google Earth). 
más externa y de gran influencia marina. Las estaciones de Tapera de López y Arroyo San Clemente corresponden a los canales internos al sur de Punta Rasa (Figura 1).

\section{Colecta de muestras y análisis estadísticos}

En cada estación se colectaron 24 muestras al azar utilizando un tubo de PVC de $12 \mathrm{~cm}$ de diámetro y $30 \mathrm{~cm}$ de longitud total. Cada muestra estuvo constituida por un cilindro de sedimento de 20 $\mathrm{cm}$ de altura, el que fue separado en dos niveles (superior e inferior) de $10 \mathrm{~cm}$ cada uno. Los organismos fueron separados con tamices de $0.5 \mathrm{~mm}$ de tamaño de malla y conservados en formaldehído neutralizado al $10 \%$ para su posterior clasificación y conteo. Para cada especie de poliqueto se determinó la Frecuencia de Ocurrencia Porcentual (FO\%, el porcentaje en que cada especie de poliqueto está presente respecto al total de muestras analizadas) y Abundancia Numérica Porcentual (AN\%, número de ejemplares de cada especie de poliqueto dividido por el total de poliquetos encontrados expresado en porcentaje).

\section{Procesamiento y caracterización del sedimento}

Se extrajeron muestras adicionales para realizar análisis sedimentarios. Una vez eliminada la totalidad del agua intersticial se procedió al cuarteo hasta llegar a submuestras que pesaron entre 20 y $100 \mathrm{~g}$ y que fueron desagregadas de forma manual con mortero de porcelana. El análisis granulométrico se realizó por tamizado según el método de Roy Ingram, con series de tamices a $1 / 2$ grado phi (según escala Udden Wentworth) en tamizadora tipo Rottap (Carver 1971). Se separó el sedimento en tres fracciones granulométricas: psefita, psamita (discriminando tres categorías: gruesa, media, fina) y pelita (incluyendo al limo y a la arcilla).

\section{Análisis estadísticos}

Las comparaciones sedimentológicas entre las estaciones de muestreo fueron realizadas por medio de un análisis de cluster de distancias euclideanas y una distribución triangular de composición da cada una de las muestras. Las diferencias en la densidad de poliquetos entre las estaciones y niveles fueron determinadas por análisis de varianza (ANOVA) de una vía. La normalidad de los datos fue evaluada por el test de Kolmogorov-Smirnov, mientras que los análisis de homogeneidad de varianzas fueron hechos con un test de Levene (Zar 1984). Cuando los datos no cumplían dichos supuestos se realizaron análisis no paramétricos.

La estructura de las asociaciones fue evaluada por medio de un análisis multivariado permutacional de la varianza (PERMANOVA) (Anderson 2001) con los datos transformados a raíz cuadrada y el índice de similaridad de Bray-Curtis con 9999 permutaciones. El diseño del PERMANOVA estuvo basado en 2 factores fijos: áreas (o estaciones) y nivel (superior e inferior). Se realizó un análisis de dispersión (PERMDISP) para determinar si la diferencia entre factores se debe a la dispersión dentro de los grupos.

\section{Resultados}

\section{Características sedimentológicas}

La fracción de sedimento dominante en las tres estaciones de las planicies del intermareal del sur de la Bahía Samborombón fue la arena fina. Las estaciones de Punta Rasa y Tapera de López estuvieron dominadas por arena fina (promedio $=80.85 \%$ y $81.96 \%$, respectivamente), con menores porcentajes de arena media y gruesa, psefitas, e insignificancia de pelitas (limo-arcilla) (Tabla 1). La estación Arroyo San Clemente presentó claras diferencias respecto a las otras dos, con dominancia de arena fina, pero en menor porcentaje respecto a las otras dos estaciones (promedio $=61.28 \%$ ), sin embargo, la principal diferencia fue el incremento del porcentaje de pelitas (promedio $=38.46 \%$ ). Las semejanzas entre Punta Rasa y Tapera de López fueron corroboradas por el dendrograma de distancias euclidianas y el triángulo de composición sedimentológica (Figura 2a y 2b).

\section{Asociaciones de poliquetos}

Fueron identificados cuatro especies de poliquetos en un total de 2045 individuos contabilizados (Laeonereis culveri: $\mathrm{n}=765$; Heteromastus similis: $\mathrm{n}=1227$; Alitta succinea:

Tabla 1. Características sedimentológicas de las tres estaciones de muestreo en el sur de la Bahía Samborombón, Argentina.

Table 1. Sedimentological characteristics of the three sampling stations at south of Bahía Samborombón, Argentina.

\begin{tabular}{lccc}
\hline Variable & Punta Rasa & Tapera de López & Arroyo San Clemente \\
\hline N & 48 & 48 & 48 \\
Psefita\% & $7.39(0.48-15.65)$ & $4.3(0.00-10.45)$ & $0.04(0.00-0.14)$ \\
Psamita & & & \\
Gruesa\% & $6.14(1.11-11.29)$ & $4.34(1.70-11.88)$ & $0.18(0.06-0.38)$ \\
Media\% & $5.43(1.11-13.32)$ & $8.05(0.48-36.07)$ & $0.05(0.01-0.28)$ \\
Fina\% & $80.85(51.58-91.88)$ & $81.96(62.87-95.61)$ & $61.28(44.29-72.49)$ \\
Pelita\% & $0.19(0.11-0.42)$ & $1.35(0.58-3.81)$ & $38.46(17.24-43.34)$ \\
\hline
\end{tabular}


a)

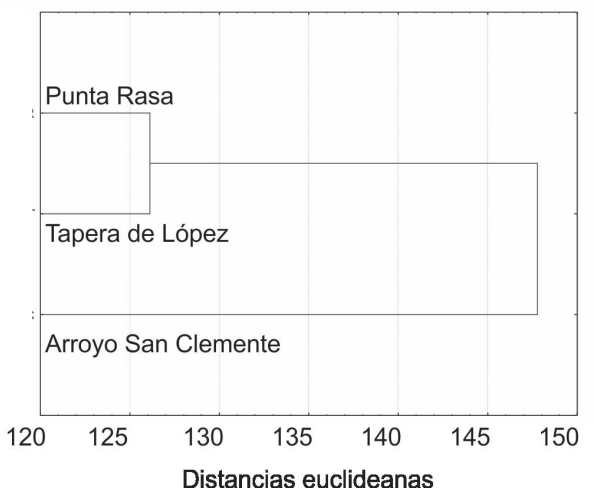

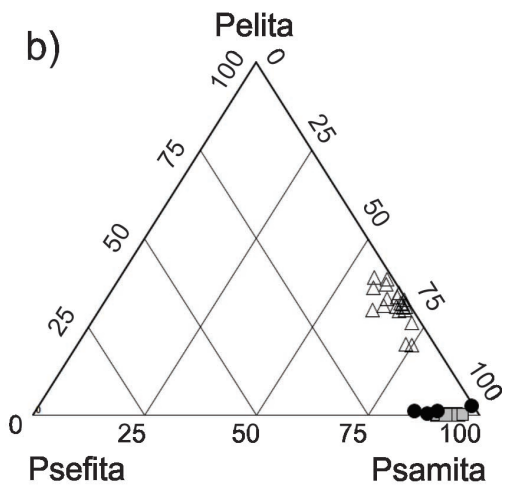

Figura 2. Dendrograma basado en distancias euclidianas medias (a) y triángulo de composición sedimentológica (b) para las tres estaciones de muestreo realizadas en el sur de la Bahía Samborombón (Argentina): $\triangle$ (Arroyo San Clemente), : Tapera de López y $\square$ : Punta Rasa.

Figure 2. Dendrogram based on mean euclidean distances (a) and sedimentological composition triangle (b) for the three sampling stations at south of Samborombón Bay (Argentina): $\triangle$ (Arroyo San Clemente), $\bullet$ : Tapera de López and $\square$ : Punta Rasa.

$\mathrm{n}=48$ y Polydora cornuta: $\mathrm{n}=5)$. Para las 4 especies combinadas y tres estaciones, la densidad promedio general en el área de estudio fue de 314 individuos $/ \mathrm{m}^{2}$. La especie más frecuente fue L. culveri $(\mathrm{FO}=72.9 \%)$, mientras que la más abundante fue $H$. similis (AN=72.4\%). Alitta succinea y P. cornuta fueron raros, con escasos valores de ocurrencia y bajos valores de abundancia (Figura 3a).

\section{Estaciones y niveles}

De las cuatro especies de poliquetos identificadas, $L$. culvieri, $H$. similis y $P$. cornuta estuvieron presentes en las tres estaciones muestreadas, mientras que $A$. succinea no fue observada en la estación Arroyo San Clemente. Punta Rasa y Tapera de López mostraron una abundancia y ocurrencia de poliquetos similar, con dominancia de L. culveri y $H$. similis (Figura 3b y 3c). Punta Rasa mostró la mayor densidad promedio de poliquetos en general con 453 individuos $/ \mathrm{m}^{2}$, seguido por Tapera de López con una densidad promedio de 397 individuos $/ \mathrm{m}^{2}$. La estación Arroyo San Clemente mostró la menor densidad promedio de poliquetos con 91 individuos $/ \mathrm{m}^{2}$, con dominancia de L. culveri y escasa presencia de $H$. similis y $P$. cornuta (Figura $3 \mathrm{~d}$ ).

Se observaron diferencias significativas en la densidad específica promedio por estaciones demuestreo para H. similis (Kruskal-Wallis test: $\left.\mathrm{H}_{(2144)}=45.45, P<0.01\right)$ y A. succinea (KruskalWallis test: $\left.\mathrm{H}_{(2,144)}=13.99, P<0.01\right)$, siendo más abundantes en Punta Rasa y Tapera de López (Tabla 2). No fueron observadas diferencias significativas en la densidad promedio de $L$. culveri (Kruskal-Wallis test: $\mathrm{H}(2,144)=1.69$, $\mathrm{P}=0.43)$ y $P$. cornuta (Kruskal-Wallis test:
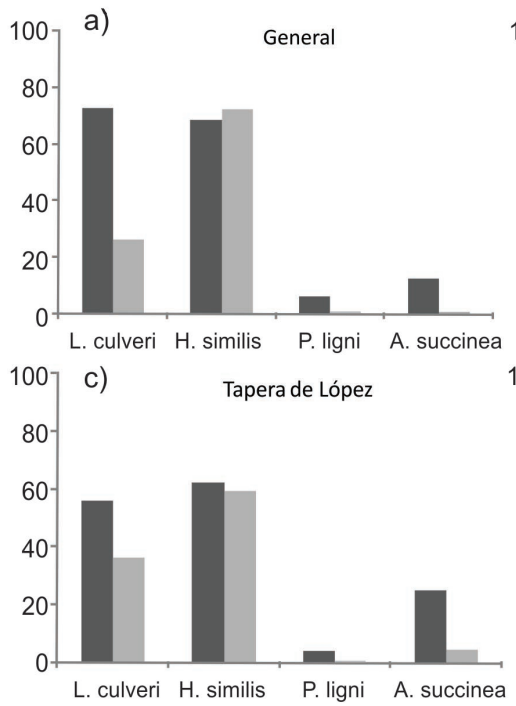
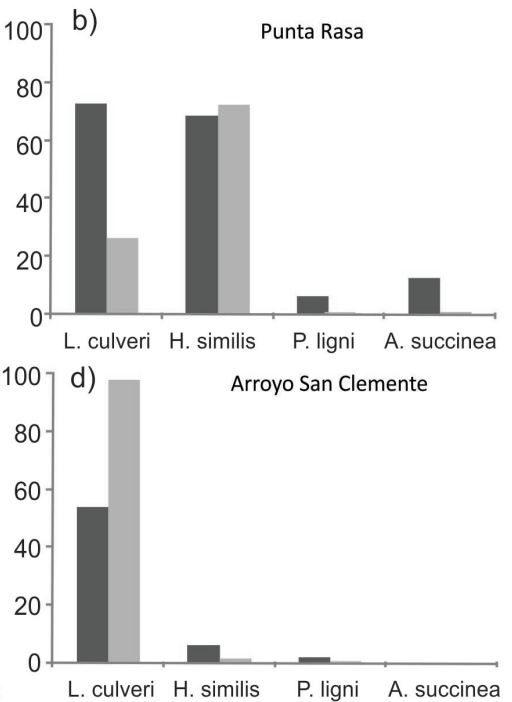

Figura 3. Frecuencia de ocurrencia (gris oscuro) y abundancia numérica (gris claro) porcentuales para las cuatro especies de poliquetos identificadas en: a) el sur de la Bahía Samborombón, b) Punta Rasa, c) Tapera de López y d) Arroyo San Clemente.

Figure 3. Frequency of occurrence (dark gray) and numerical abundance (light gray) percentage for all four polychaete species identified in: a) the south of Samborombón Bay, b) Punta Rasa, c) Tapera López and d) Arroyo San Clemente. 
Tabla 2. Información descriptiva del presente estudio: estaciones de muestreo, número de especies identificadas y densidades (individuos $/ \mathrm{m}^{2}$ ) para las cuatro especies de poliquetos identificadas en el sur de la Bahía Samborombón, Argentina.

Table 2. Descriptive information of the present study: sampling stations, species number recorded and densities (individual $/ \mathrm{m}^{2}$ ) for all four polychaete species identified in south of the Samborombón Bay, Argentina.

\begin{tabular}{|c|c|c|}
\hline \multicolumn{2}{|c|}{ Estaciones estudiadas } & 3 \\
\hline $\mathrm{N}^{\circ}$ especies poliquet & s identificadas & $\begin{array}{c}4 \\
\text { Densidad } \\
\text { (individuos } / \mathrm{m}^{2} \text { ) } \\
314\end{array}$ \\
\hline \multicolumn{3}{|c|}{ Densidad general poliquetos por estación } \\
\hline & Punta Rasa & 453 \\
\hline & $\begin{array}{l}\text { Tapera de López } \\
\text { Arroyo San } \\
\text { Clemente }\end{array}$ & $\begin{array}{c}397 \\
91\end{array}$ \\
\hline \multicolumn{3}{|c|}{ Densidad específica por estación } \\
\hline \multirow[t]{3}{*}{ Laeonereis culveri } & Punta Rasa & 475 \\
\hline & Tapera de López & 575 \\
\hline & $\begin{array}{l}\text { Arroyo San } \\
\text { Clemente }\end{array}$ & 359 \\
\hline \multirow[t]{3}{*}{ Heteromastus similis } & Punta Rasa & 1313 \\
\hline & Tapera de López & 941 \\
\hline & $\begin{array}{l}\text { Arroyo San } \\
\text { Clemente }\end{array}$ & 6 \\
\hline \multirow[t]{3}{*}{ Polydora cornuta } & Punta Rasa & 6 \\
\hline & Tapera de López & 2 \\
\hline & $\begin{array}{l}\text { Arroyo San } \\
\text { Clemente }\end{array}$ & 2 \\
\hline \multirow[t]{3}{*}{ Alitta succinea } & Punta Rasa & 18 \\
\hline & Tapera de López & 70 \\
\hline & $\begin{array}{l}\text { Arroyo San } \\
\text { Clemente }\end{array}$ & 0 \\
\hline
\end{tabular}

$\mathrm{H}(2,144)=1.64, \mathrm{P}=0.44)$ en las estaciones de muestreo. Las densidades específicas en cada estación de muestreo estimadas fueron menores a aquellas registradas en estudios llevados a cabo durante la década del noventa. Estas diferencias, sin embargo, no pudieron ser corroboradas estadísticamente por no disponer de los datos originales.

Las cuatro especies de poliquetos estuvieron presentes en el nivel superior, mientras que $P$. cornuta y A. succinea no fueron observadas en el nivel inferior. La densidad promedio de poliquetos para el nivel superior de las estaciones Punta Rasa y Tapera de López fue significativamente superior sólo para $L$. culveri y $H$. similis $(P<0.01)$, y no se observaron diferencias entre las especies menos abundantes. Para Arroyo San Clemente sólo se observaron diferencias entre niveles para L. culveri, siendo significativamente mayor su densidad en el nivel superior A $(P<0.01)$.

Las variaciones entre estaciones y niveles observadas en las asociaciones de poliquetos fueron corroboradas por el PERMANOVA, cuyo principal componente de variación estuvo dado por los niveles $(P<0.01$; varianza: $36 \%)$, seguido por las estaciones $(P<0.01$; varianza: $36 \%$ ).

\section{DisCUSIÓN}

Gran parte de los sedimentos de la Bahía de Samborombón es aportada por el Río de la Plata, que transporta aquellos provenientes del Río Paraná. La otra fuente de sedimentos es de origen continental, aportada por la cuenca del Río Salado y los canales de drenaje (escurrimiento) de este sector de la Provincia de Buenos Aires. Existe un aporte sedimentario de origen marino en la zona cercana al frente marítimo del estuario (Simionato et al. 2011).

Sibien puedeexistir un esquema sedimentario a gran escala que tipifique este sector del estuario, los estudios realizados en la zona de San Clemente del Tuyú indican que pueden presentarse diferencias en la composición sedimentaria de los fondos, incluso entre estaciones relativamente próximas entre sí. En el presente estudio, cada estación de muestreo presentó un esquema sedimentario particular con un grado variable de similitud. La estación de Punta Rasa presentó mayor similitud con Tapera de López debido a su gran representación de la fracción de arena fina. En la estación Arroyo San Clemente fue importante la fracción sedimentaria más fina, debido a que es la zona más protegida de los vientos y donde la turbulencia del agua es mínima y la resuspensión de los sedimentos en la columna de agua es mayor (Giménez et al. 2006). La composición sedimentaria de la estación Tapera de Lópezy Punta Rasa indica la influencia marina, ya que las psefitas, la arena gruesa y la mediana suelen ser componentes importantes de las arenas de playas marinas al sur de Punta Rasa (Ieno \& Bastida 1998; Martin et al. 2004). Estas particularidades sedimentarias que exhiben las estaciones hacen que puedan correlacionarse con la composición de las asociaciones de poliquetos presentes, como se detalla a continuación.

Fueron identificadas cuatro especies de poliquetos: Laeonereis culveri, Heteromastus similis, Alitta succinea y Polydora cornuta, las que fueron identificadas previamente en la zona (Ieno \& Bastida 1998; Martin et al. 2004). Nepthys fluviatilis, Capitella capitata y Scolecolepides sp., poco abundantes en la región, no fueron encontradas en este estudio. Al igual que en trabajos previos (Ieno \& Bastida 1998; Martin et al. 2004), los poliquetos dominantes 
fueron $H$. similis y L. culveri, lo que sugiere una estabilidad en la presencia de especies en las últimas décadas.

Los primeros estudios faunísticos del macrobentos realizados en el estuario de la Bahía Samborombón corresponde a dos trabajos durante 1993-1994 (Ieno \& Bastida 1998) y 1998-1999 (Martin et al. 2004). En ambos se destaca una baja riqueza específica y una dominancia de poliquetos como patrón característico no sólo en esta área de estudio, sino también de otros ambientes mixohalinos de la provincia de Buenos Aires. Asimismo, cabe destacar que las abundancias de poliquetos para la época de muestreo se corresponden con la más baja de su ciclo anual (Ieno \& Bastida 1998; Martin et al. 2004) debido a que en la Bahía de Samborombón son un recurso trófico fundamental de las aves playeras (Morrison et al. 1989), tanto locales como migratorias, además de varias especies de peces de interés comercial como la corvina rubia Micropogonias furnieri y la corvina negra Pogonias cromis (Lasta 1995). Numerosas aves migratorias basan su dieta fundamentalmente sobre poliquetos y pequeños moluscos (Botto et al. 1998; Ieno et al. 2004) y llegan a la zona para la primavera y verano del Hemisferio Sur. Según Morrison et al. (1989) los censos de aves efectuados en la Bahía Samborombón representan entre $63 \%$ y $75 \%$ de las aves que permanecen en el verano austral en la Argentina, lo cual sugiere una depredación intensa. Es posible que los resultados de este trabajo se correspondan a la etapa inmediatamente posterior a una depredación estival intensiva de estos organismos bentónicos, proceso que se ha venido observando en los últimos 20 años (Ieno \& Bastida 1998; Martin et al. 2004). Durante los siguientes meses de otoño e invierno la densidad de poliquetos sufre una notable recuperación y alcanza los niveles máximos previo a la primavera siguiente (Martin et al. 2004). Esta dinámica en particular es frecuente en comunidades bentónicas de estuarios y lagunas costeras ya que experimentan un altísimo dinamismo ambiental sujeto a variaciones temporales y a factores como la salinidad, temperatura, intrusión de agua, etc. (Meerhoff et al. 2013).

Las características sedimentológicas son los factores de pequeña escala que más afecta la distribución de los organismos, según los estudios sedimentológicos de la zona estudiada (Ieno \& Bastida 1998; Martin et al. 2004; Meerhoff et al. 2013) y en otros ambientes costeros de la región (Giménez et al. 2014). En relación a lo expuesto, las diferencias en las características del sedimento de las estaciones estudiadas también fueron observadas en la estructura de las asociaciones de poliquetos. Cabe señalar que Punta Rasa y Tapera de López presentaron una composición y abundancia similar de poliquetos, con una dominancia de $H$. similis. Sin embargo, resultados previos han reconocido a L. culveri como poliqueto dominante (Martin et al. 2004). Las asociaciones de poliquetos observados en Arroyo San Clemente resultaron diferentes a los otros dos ambientes estudiados con una dominancia casi exclusiva de L. culveri, correspondiéndose con estudios previos (Ieno \& Bastida 1998; Martin et al. 2004), sugiriendo, de este modo, una presencia más estable en los canales internos de la Bahía que en zonas más expuestas de la costa como Tapera de López y principalmente Punta Rasa. Alitta succinea estuvo ausente en esta estación, posiblemente por ser un poliqueto con mayor afinidad a ambientes marinos (Martin 2002; Giménez et al. 2014).

Por otra parte, y en relación a los niveles verticales del sedimento, sólo $H$. similis y L. culveri fueron observados en los niveles superior einferior. Ambas estuvieron presentes en mayor densidad en el nivel superior para las tres estaciones estudiadas, a diferencia de lo observado por Martin et al. (2004), quienes encontraron una mayor afinidad de $H$. similis y A. succinea en el nivel inferior.

El presente estudio describe la composición de poliquetos bentónicos de la Bahía de Samborombón, y las variaciones relacionadas al tipo de sedimento. Considerando el potencial de los poliquetos como organismos indicadores de impacto ambiental, sugerimos que los monitoreos bentónicos en dicha zona deberían desarrollarse en el futuro cada 5 ó 10 años, teniendo en consideración la variación estacional, a los efectos de tener un control ambiental eficiente a través de estas especies de poliquetos.

Agradecimientos: Los autores agradecen la colaboración del Oceanario Mundo Marino y especialmente al entrenador Jorge Rebollo por la logística durante la extracción de las muestras de sedimentos. También agradecemos la planificación y asesoramiento de la Dra. Elena Ieno durante el presente estudio.

\section{BiBLIOGRAFÍA}

Anderson, MJ. 2001. A new method for non-parametric multivariate analysis of variance. Austral Ecol., 26:32-46. Botto, F; O Iribarne; M Martínez; K Delhey \& M Carrete. 
1998. The effect of migratory shorebirds on the benthic species of three southwestern Atlantic Argentinean estuaries. Estuaries, 21(48):700-709.

Botto, F; E Gaitán; H Mianzan; M Acha; D Giberto; et AL. 2011. Origin of resources and trophic pathways in a large SW Atlantic estuary: An evaluation using stable isotopes. Estuarine, Coastal and Shelf Science, 92:70-77.

CARVER, RE. 1971. Procedures in Sedimentary Petrology. Wiley-Interscience, New York.

GiménEZ, L; C Dimitriadis; A Carranza; A Borthagaray \& M RodRíGUEZ. 2006. Unravelling the complex structure of a benthic community: A multiscale-multianalytical approach to an estuarine sandflat. Est. Coast. Shelf Sci., 68:462-472.

Giménez, L; N Venturini; N Kandratavicius; M Hutton; A LAFRANCONI; ET AL. 2014. Macrofaunal patterns and animal-sediment relationships in Uruguayan estuaries and coastal lagoons (Atlantic coast of South America). J. Sea Res., 87:46-55.

IENO, E \& R BASTIDA. 1998. Spatial and temporal patterns in coastal macrobenthos of Samborombon Bay, Argentina: a case study of very low diversity. Estuaries, 21(4):690699.

IenO, E \& R ELíAs. 1995. Heteromastus similis Southern, 1921 (Polychaeta: Capitellidae) in Mar Chiquita brackish coastal lagoon, Argentina. Nerítica, 9:23-32.

Ieno, E, D Alemany, D Blanco, \& R Bastida. 2004. Prey size selection of Red Knot (Calidris canutus) feeding on mud snail (Litoridina australis) during northward migration at Punta Rasa (Buenos Aires Province, Argentina). Waterbirds, 27(4):493-498.

LASTA, CA. 1995. La Bahía Samborombón: zona de desove y cría de peces. Tesis Doctoral. Universidad Nacional de La Plata, Argentina.

López Gappa, J; A Tablado; MC Fonalleras \& ML ADAMI. 2001. Temporal and spatial patterns of annelid populations in intertidal sediments of the Quequén Grande estuary (Argentina). Hydrobiologia, 455:61-69.

MARTIN, JP. 2002. Biología y ecología de poliquetos de ambientes mixohalinos de la provincia de Buenos Aires. Tesis Doctoral. Universidad Nacional de Mar del Plata, Argentina.
Martin, JP; R Bastida \& M Trassens. 2004. Polychaete assemblages of intertidial flats of Bahía Samborombón (La Plata River Estuary-Argentina). Thalassas, 20(2): 39-53.

Meerhoff, E; L Rodríguez-Gallego; L Giménez; P Muniz \& D Conde. 2013. Spatial patterns of macrofaunal community structure in coastal lagoons of Uruguay. Mar. Ecol. Prog. Ser., 492:97-110.

Morrison, RIG; P CANEVARI \& K Ross. 1989. Argentina. Pp. 218-246 en: Morrison, RIG \& RK Ross (eds.). Atlas of Neartic shorebirds on the coast of South America. Vol. 2. Canadian Wildlife Service Special Publication.

Muniz, P; M Hutton; N Kandratavicius; A Lafranconi; E Brugnoli; et AL. 2012. Performance of biotic indices in naturally stressed estuarine environments on the Southwestern Atlantic coast (Uruguay): A multiple scale approach. Ecol. Indic., 19:89-97.

Olivier, SR; A Escofet; P Penchaszadeh \& JM Orenzanz. 1972. Estudios ecológicos en la región estuarial de Mar Chiquita (Buenos Aires, Argentina). I. Las comunidades bentónicas. Anales de la Sociedad Científica Argentina, 193: 237-262.

RingueLET, RA. 1962. Ecología acuática continental. Editorial Eudeba.

Rodríguez, D; L Rivero \& R Bastida. 2002. Feeding ecology of the franciscana (Pontoporia blainvillei) in estuarine and marine waters of northern Argentina. LAJAM, 1(1):7794.

SChwindt, E \& O Iribarne. 2000. Settlement sites, survival and effects on benthos of an introduced ref-building polychaete in a SW Atlantic coastal lagoon. Bull. Mar. Sci., 67(1):73-82.

Simionato, CH; D Moreira \& M Fossatti. 2011. Estudio de la dinámica hidro-sedimentológica del Rio de La Plata: Observación y modelación numérica de los sedimentos finos. Proyecto FREPLATA LA 99/G31. Pp. 109.

URIEN, CM. 1966. Distribución de los sedimentos en el Río de la Plata Superior, Tirada Aparte del Boletín del Servicio de Hidrografía Naval, 3:197-203.

ZAR, WJ. 1984. Biostatistical analysis. Prentice Hall Inc. New Jersey. 